\title{
JOGOS E MATERIAIS MANIPULÁVEIS PRODUZIDOS POR ALUNOS DO IFBA, CAMPUS DE EUNÁPOLIS
}

\author{
Marcio Antonio Souza Paim ${ }^{1}$
}

\begin{abstract}
RESUMO
O objetivo deste trabalho é mostrar exemplos de jogos e materiais manipuláveis criados por alunos da $1^{\mathrm{a}}$ série do ensino médio do IFBA, campus de Eunápolis, para auxiliar no processo de ensino e aprendizagem da matemática. Sob a supervisão do professor da disciplina, três grupos de cinco estudantes do Curso Técnico em Edificações do Instituto Federal da Bahia, localizado na cidade de Eunápolis, no extremo sul do mesmo estado, construíram três tipos de subsídios de matemática, na forma de jogos e materiais manipuláveis, para o Laboratório de Matemática da Instituição. Espera-se que o material manipulável, quando associado ao jogo, contribua para o desenvolvimento da criatividade dos estudantes e compreensão de conteúdos matemáticos, fortalecendo o interesse pela pesquisa em matemática.
\end{abstract}

Palavras-chave: Materiais Manipuláveis. Jogos. Ensino e Aprendizagem da Matemática.

\section{GAMES AND MANIPULATIVES PRODUCED BY STUDENTS IFBA, CAMPUS EUNÁPOLIS}

\begin{abstract}
The objective of this work is to show examples of manipulative materials and games created by IFBA high school 1st year students, Eunápolis Campus, to assist in teaching and learning of mathematics process. Under the supervision of the subject teacher, tree groups of five students of the building technical course built tree types of math subsidies in games form and manipulative materials for the Mathematics Laboratory Facility. It is expected that the manipulable material when associated with games, contribute to the development of student creativity and understanding of mathematical content, strengthening the interest in research in mathematics.
\end{abstract}

Keywords: Manipulatives. Games. Teaching and Learning of Mathematics.

\footnotetext{
${ }^{1}$ Graduado em licenciatura em Matemática pela Universidade Federal da Bahia (UFB). Atualmente é professor de Matemática do Ensino Básico Técnico e Tecnológico do IFBA -Campus de Santo Amaro Bahia. E-mail: maspaim@hotmail.com.
}

ForSci.: r. cient. IFMG campus Formiga, Formiga, v. 2, n. 1, p. 113-123 jan./jun. 2014 


\title{
1 INTRODUÇÃO
}

A Educação Matemática tem se mostrado presente nas escolas e tem recebido certa atenção em nosso país através de ações que visam à melhoria do ensino da matemática. Assim acontece porque, entre outras questões, as rápidas alterações sociais têm exigido da escola um repensar de suas práticas pedagógicas (LOPES, 2004).

Lorenzato (2009, p. 40) afirma:

\begin{abstract}
As novas demandas sociais educativas apontam para a necessidade de um ensino voltado para a promoção do desenvolvimento da autonomia intelectual, criatividade e capacidade de ação, reflexão e crítica pelo aluno. Para tanto, faz-se necessário a introdução da aprendizagem de novos conteúdos de conhecimentos e de metodologias que, baseadas na concepção de que o aluno deve ser o centro do processo de ensinoaprendizagem, reconheça, identifique e considere seus conhecimentos prévios como ponto de partida e o prepare para realizar-se como cidadão em uma sociedade submetida a constantes mudanças.
\end{abstract}

A exigência da aprendizagem matemática nas escolas vem crescendo na medida em que a tecnologia e a sociedade abrem espaço para novos cenários de pesquisas educacionais que geram diferentes reflexões. Isso vem comprovando a grande evolução dos estudos nacionais nesta área ao longo dos anos: Borba e Penteado (2010) compreendem a informática como uma das principais tendências em Educação Matemática, enquanto Santana (2010) faz uso de materiais didáticos para o estudo do Campo Conceitual Aditivo de determinados estudantes.

Vários grupos de pesquisa estão sendo criados por pesquisadores que discutem sobre as práticas de ensino e aprendizagem. É possível perceber o interesse na criação de novas estratégias e técnicas de ensino nas quais os alunos sejam sujeitos de transformação da sua própria realidade ao longo da carreira escolar. Faz-se mais do que necessário que o que se ensina valha a pena (FORQUIN, 1993).

A matemática também pode ser explorada por meio de jogos e materiais manipuláveis; porém, não é comum existirem nas escolas materiais concretos para o ensino da matemática. Muitos citam que a justificativa para a não utilização está na filosofia de alguns professores e até na política escolar. Segundo Lorenzato (2009), há mitos e preconceitos que retardam e impedem o uso destes materiais na sala de aula.

O fato é que, para muitos, esses materiais representam uma conexão entre o mundo abstrato da matemática e o seu mundo físico, prático e real. Para Bravo (1995), o uso desses recursos pelo professor na sala de aula é mais do que necessário. 
Materiais manipuláveis, segundo a definição de Reys (1971, apud MATOS; SERRAZINA, 1996, p. 193), são “[...] objetos ou coisas que o aluno é capaz de sentir, tocar, manipular e movimentar. Podem ser objetos reais que têm aplicação no dia a dia ou podem ser objetos que são usados para representar uma ideia".

Por sua vez, Lorenzato (2009) revela os materiais manipuláveis como qualquer instrumento útil ao processo de ensino-aprendizagem. Por isso, há um cuidado no correto uso destes materiais pelo professor, devendo ser de forma planejada e articulada, a fim de que funcionem como uma primeira forma de representação de conceitos (PAIS, 1996) e que os próprios conceitos tenham conexões com as aplicações teóricas da disciplina. Além disso, atividades com recursos manipuláveis não podem estar desvinculadas da existência de uma intuição e de um nível de racionalidade.

Sendo assim, o presente trabalho teve como objetivo mostrar a produção de três grupos de cinco alunos do Ensino Básico Técnico e Tecnológico em Edificações do Instituto Federal de Educação, Ciência e Tecnologia da Bahia, do campus de Eunápolis, extremo sul do estado da Bahia, entre os anos de 2010 e 2011. Eles produziram alguns jogos e materiais manipuláveis para o ensino da matemática, com o objetivo de enriquecer o Laboratório de Ensino da Matemática (LEM) da mesma instituição e contribuir para a obtenção de conhecimentos matemáticos de professores e alunos de séries diferentes. A criação foi coletiva e os participantes utilizaram materiais recicláveis para a realização das tarefas.

Vale ressaltar que os materiais foram feitos tendo como base os dominós, o jogo da memória, os pentaminós e as pirâmides. Os assuntos abordados foram as noções básicas de equações exponenciais e logarítmicas, a potenciação, os números decimais e as sequências numéricas. Todos os assuntos foram, respectivamente, referentes às turmas de nível médio dos cursos integrados da instituição. Seguem as criações de cada grupo.

\section{O DOMINÓ DE LOGARITMOS}

O dominó é um jogo bem popular muito visto em diversas comunidades e é frequentemente jogado por pessoas de várias idades. Nos intervalos escolares e nas horas vagas, é fácil observar que muitos jogam pelo simples prazer de "contar" as suas peças, 28 no total, e que, geralmente, são distribuídas para quatro jogadores com sete peças para cada.

A face da peça de um dominó é retangular, dividida em dois quadrados, e, em cada quadrado, há a caracterização de números naturais que variam de 0 a 6 . Do ponto de vista 
numérico, uma peça de dominó representa um par de números naturais, ou seja, $(4,2),(0,1)$, $(5,4), \ldots$, são exemplos de três pares descritos, respectivamente, em três peças.

Sendo assim, a equipe responsável pela criação do jogo resolveu se apropriar do conteúdo matemático dos logaritmos para escrever os pares das peças. Algumas peças foram construídas pelos alunos para fins de resolução de uma expressão logarítmica do tipo $\log _{a} b$, com $0<a \neq 1$ e $b>0, a$ e $b$ sendo números naturais.

Uma peça formada pelas expressões logarítmicas $\log _{3} 27$ e $\log _{4} 16$, cujos valores são numericamente iguais a 3 e 2 , é formada pelo par $\left(\log _{3} 27, \log _{4} 16\right)$ e corresponde a $(3,2)$, assim como $\left(3, \log _{2} 64\right)$ é igual a $(3,6)$. O exemplo a seguir ilustra esse fato através da definição de logaritmos: $\log _{a} b=x \Leftrightarrow a^{x}=b$ :

$$
\begin{aligned}
& \log _{3} 27=x \Rightarrow 3^{x}=27 \Rightarrow 3^{x}=3^{3} \Rightarrow x=3 \\
& \log _{4} 16=y \Rightarrow 4^{y}=16 \Rightarrow 4^{y}=4^{2} \Rightarrow y=2 \\
& \log _{2} 64=z \Rightarrow 2^{z}=64 \Rightarrow 2^{z}=2^{6} \Rightarrow z=6
\end{aligned}
$$

Cada peça foi feita com recorte de papel-cartão cor-de-rosa de mesma medida, com o objetivo de encaixar as peças de acordo com o valor correspondente aos seus logaritmos:

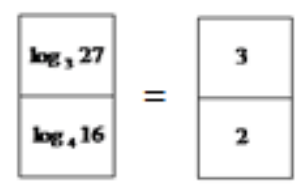

Figura 1 - Peças correspondentes Fonte: Elaborada pelo autor

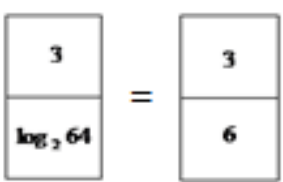

E assim, sucessivamente, até que os participantes efetuassem os cálculos correspondentes. Uma configuração possível para o dominó também poderia ser representada por:

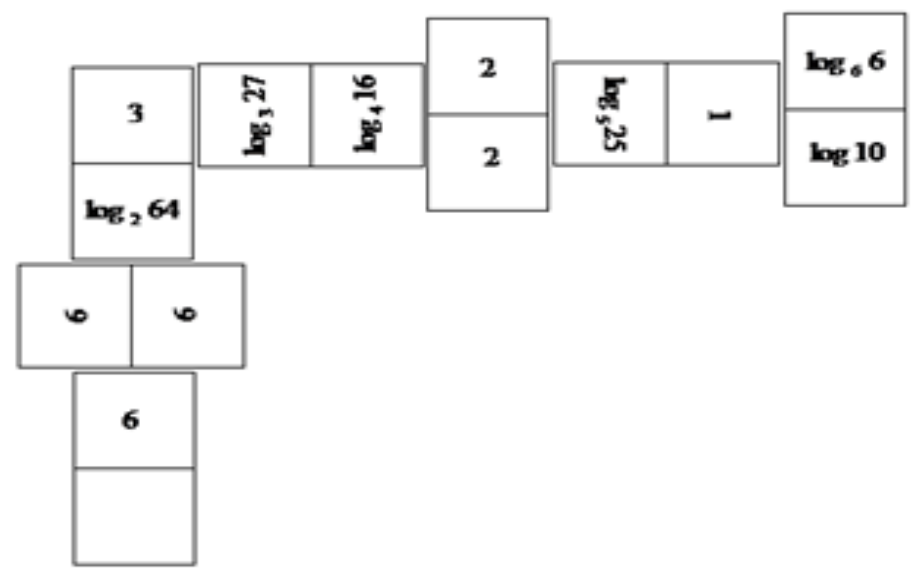

Figura 2 - Dominó de logaritmos

Fonte: Elaborada pelo autor

ForSci.: r. cient. IFMG campus Formiga, Formiga, v. 2, n. 1, p. 113-123 jan./jun. 2014 
Os erros cometidos pelos alunos serviram de base para a busca das respostas corretas. A ideia do 'é errando que se aprende' foi constante na criação dos jogos, visto que algumas peças foram produzidas mediante dúvidas dos alunos e provocaram questionamentos inesperados que posteriormente foram sanados com a ajuda do professor.
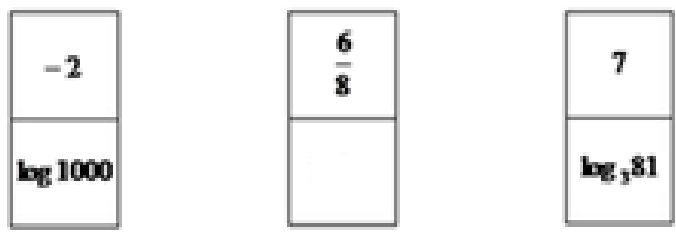

Figura 3 - Peças sem significado

Fonte: Elaborada pelo autor

Na Figura 3, a primeira peça representa o par $(-2,3)$, onde o primeiro elemento é um número inteiro não positivo e que não faz parte do conjunto $D=\{0,1,2,3,4,5,6\}$ dos números que representam as peças do dominó. Já a segunda mostra que a fração $\frac{6}{8}$ é uma parte do inteiro - elemento diferente dos elementos do mesmo conjunto $D$. A última sugere o par $(7,4)$ com $7 \notin D$.

\section{O JOGO DA MEMÓRIA}

O jogo inicia com todas as cartas com as faces viradas para baixo, o jogador escolhe $\mathrm{e}$ vira duas cartas; se elas forem iguais, são retiradas; caso contrário, são novamente viradas. Mais uma vez, as figuras utilizadas foram expressões logarítmicas e exponenciais com a mesma simplicidade das do dominó.

O material de confecção feito pelos alunos foi composto de uma madeira bem rígida e quadrada. Em cada face do quadrado as expressões foram escritas à mão e pintadas com tinta guache colorida. Sendo $0<a \neq 1, b>0, c>0, a, b$ e $c$ números reais, pesquisando em livros didáticos, os alunos verificaram que as propriedades básicas necessárias para a execução do jogo seriam:
i) $\log _{a} b=x \Leftrightarrow a^{x}=b$
ii) $\log _{a} a=1$
iii) $\log _{a} b^{n}=n \cdot \log _{a} b$
iv) $\log _{a} b=\frac{\log _{c} b}{\log _{c} a}$ 
Algumas peças foram justapostas com as faces voltadas para cima:
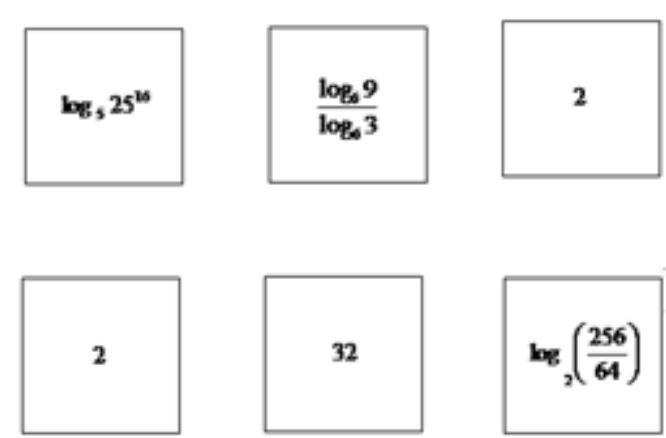

Figura 4 - Seis peças do jogo da memória Fonte: Elaborada pelo autor

Com todos os jogadores resolvendo as expressões, tivemos:

a) $\log _{5} 25^{16}=16 . \log _{5} 25=16 . \log _{5} 5^{2}=16.2 \cdot \log _{5} 5=32.1=32$;

b) $\frac{\log _{6} 9}{\log _{6} 3}=\log _{3} 9=\log _{3} 3^{2}=2 \log _{3} 3=2.1=2$;

c) $\log _{2}\left(\frac{256}{64}\right)=\log _{2} 4=\log _{2} 2^{2}=2 \cdot \log _{2} 2=2.1=2$.

Restava aos alunos associar cada peça virada para baixo ao seu valor correspondente.

\section{NÚMEROS TETRAÉDRICOS E TRIANGULARES}

Os números tetraédricos ou piramidais são uma sequência numérica de números figurados e espaciais representados por tetraedros com quatro faces compostas de triângulos equiláteros. Um número piramidal pode ser simbolizado por uma pirâmide que possui faces laterais, a face da base, as arestas e os vértices como os principais elementos de uma figura espacial. Foi proposta a construção de uma pirâmide de base e faces laterais triangulares.

Os alunos substituíram as arestas e os vértices, respectivamente, por palitos de picolé e bolinhas de isopor, fixando as pontas dos palitos nas bolinhas de isopor para dar origem a cada pirâmide. 
Exemplo:
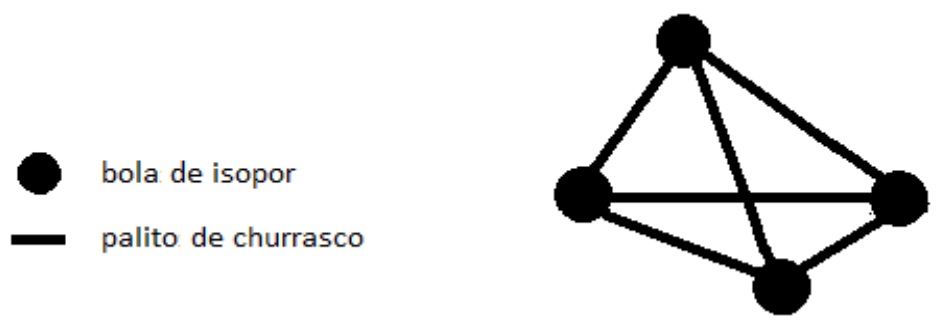

Figura 5 - Segundo número tetraédrico Fonte: Elaborada pelo autor

O objetivo foi indicar quem conseguiria formar mais rapidamente os cinco primeiros números tetraédricos. Antes dessa prática, foi mostrado que, além dos números tetraédricos, vários outros conjuntos de números eram obtidos através do Triângulo de Pascal, que relaciona números com figuras geométricas planas e espaciais. A primeira coluna do Triângulo de Pascal é a zero:

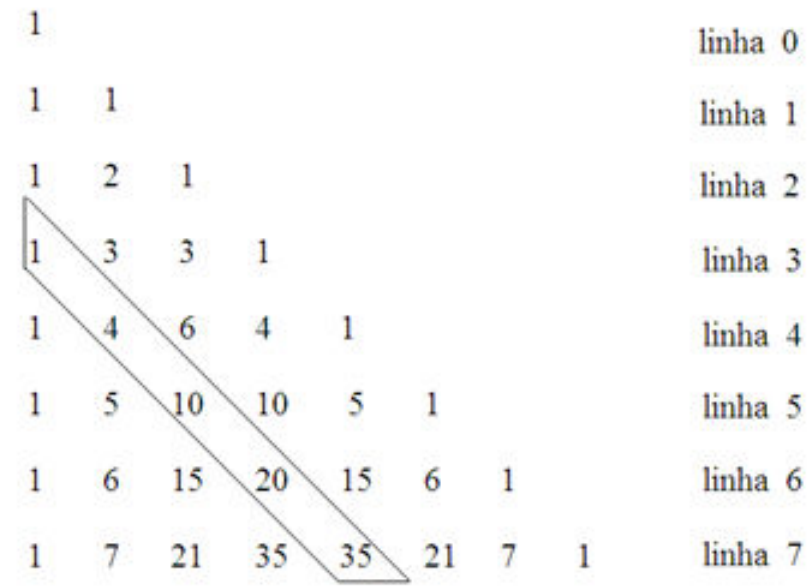

Figura 6 - Triângulo de Pascal

Fonte: Elaborada pelo autor

Os números situados na diagonal em destaque: $1,4,10,20,35, \ldots$ são os tetraédricos, enquanto que, logo acima, estão os triangulares: $1,3,6,10,15, \ldots$, os quais podem ser assim definidos:

$a_{1}=1, a_{2}=1+2=3, a_{3}=1+2+3=6, a_{4}=1+2+3=4=10, a_{5}=1+2+3+4+5=15$, $a_{6}=1+2+3+4+5+6=21, \ldots ., a_{n}=S_{n}=1+2+3+4+5+6+\ldots+n$.

$S_{n}$ é a soma dos termos de uma P.A. de razão igual a 1. Portanto, foi possível obter qualquer número triangular pela fórmula: 


$$
S_{n}=1+2+3+4+5+6+\ldots+n=\frac{\left(a_{1}+a_{n}\right) \cdot n}{2}=\frac{(n+1) \cdot n}{2}, \operatorname{com} n \in N^{*}
$$

Previamente explicado que $n !=n .(n-1) .(n-2) \ldots 3 \cdot 2.1, n \in N, n>1,0 !=1$ e $1 !=1$, enfatizou-se que estes números triangulares são também coeficientes binomiais ou números combinatórios do tipo $C_{n, p}=\left(\begin{array}{l}n \\ p\end{array}\right)=\frac{n !}{p !(n-p) !}$ situados na linha $n$ e coluna $p$ do Triângulo de Pascal. Dessa forma, foi fácil obter, por exemplo, $1=\left(\begin{array}{l}2 \\ 2\end{array}\right), 3=\left(\begin{array}{l}3 \\ 2\end{array}\right), 6=\left(\begin{array}{l}4 \\ 2\end{array}\right), 10=\left(\begin{array}{l}5 \\ 2\end{array}\right)$, e assim por diante.

Os alunos conjecturavam e calculavam de acordo com a observação do triângulo. Numa dessas constatações, a soma de números triangulares consecutivos seria um tetraédrico, de fato:

$$
\begin{aligned}
& a_{1}=1=1 \\
& a_{2}=1+3=4 \\
& a_{3}=1+3+6=10 \\
& a_{4}=1+3+6+10=20 \\
& a_{5}=1+3+6+10+15=35 \\
& a_{6}=1+3+6+10+15+21=56
\end{aligned}
$$

O cálculo para o enésimo termo estaria numa das propriedades básicas do Triângulo de Pascal em que a soma dos elementos de uma coluna, começando em seu primeiro elemento, é igual ao elemento que está avançado uma linha e uma coluna sobre a última parcela da soma (Teorema das Colunas):

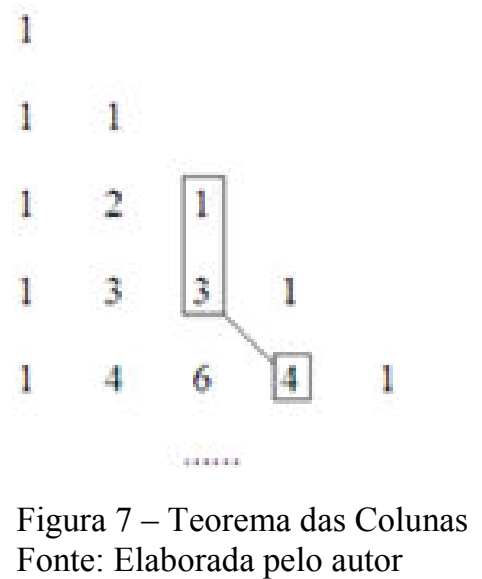


Na coluna 2 , temos a soma $1+3=4$, ou $1+3+6=10$, e assim por diante. De um modo geral, $1=\left(\begin{array}{c}1+1 \\ 2\end{array}\right)=\left(\begin{array}{l}2 \\ 2\end{array}\right), 3=\left(\begin{array}{c}2+1 \\ 2\end{array}\right)=\left(\begin{array}{l}3 \\ 2\end{array}\right), 10=\left(\begin{array}{c}3+1 \\ 2\end{array}\right)=\left(\begin{array}{l}4 \\ 2\end{array}\right), \ldots$, portanto:

$$
\begin{aligned}
& 1=\left(\begin{array}{c}
1+1 \\
2
\end{array}\right), 1 \text { parcela } \\
& 4=1+3=\left(\begin{array}{c}
1+1 \\
2
\end{array}\right)+\left(\begin{array}{c}
2+1 \\
2
\end{array}\right), 2 \text { parcelas } \\
& 10=1+3+6=\left(\begin{array}{c}
1+1 \\
2
\end{array}\right)+\left(\begin{array}{c}
2+1 \\
2
\end{array}\right)+\left(\begin{array}{c}
3+1 \\
2
\end{array}\right), 3 \text { parcelas }
\end{aligned}
$$

Para $k$ variando de 1 a $n$ parcelas, a soma $\sum_{1}^{n}\left(\begin{array}{c}k+1 \\ 2\end{array}\right)$ transforma-se imediatamente em $\left(\begin{array}{c}n+2 \\ 3\end{array}\right) p / n$ parcelas, ou seja:

$$
\left(\begin{array}{c}
1+1 \\
2
\end{array}\right)+\left(\begin{array}{c}
2+1 \\
2
\end{array}\right)+\left(\begin{array}{c}
3+1 \\
2
\end{array}\right)+\left(\begin{array}{c}
4+1 \\
2
\end{array}\right)+\ldots+\left(\begin{array}{c}
n+1 \\
2
\end{array}\right)=\left(\begin{array}{c}
n+1+1 \\
2+1
\end{array}\right)=\left(\begin{array}{c}
n+2 \\
3
\end{array}\right)
$$

Que nada mais é do que o enésimo termo tetraédrico:

$$
\left(\begin{array}{c}
n+2 \\
3
\end{array}\right)=\frac{(n+2) !}{3 ! \cdot(n+2-3) !}=\frac{(n+2) \cdot(n+1) \cdot n(n-1) !}{6 \cdot(n-1) !}=\frac{(n+2) \cdot(n+1) \cdot n}{6}
$$

Por exemplo, se $k=5$ parcelas, então o número piramidal será igual a:

$$
\left(\begin{array}{l}
2 \\
2
\end{array}\right)+\left(\begin{array}{l}
3 \\
2
\end{array}\right)+\left(\begin{array}{l}
4 \\
2
\end{array}\right)+\left(\begin{array}{l}
5 \\
2
\end{array}\right)+\left(\begin{array}{l}
6 \\
2
\end{array}\right)=\left(\begin{array}{l}
5+2 \\
2+1
\end{array}\right)=\left(\begin{array}{l}
7 \\
3
\end{array}\right)=35
$$

É bom lembrar que as demonstrações para os principais teoremas do Triângulo de Pascal e para os teoremas das colunas podem ser vistas em diversos livros de ensino médio e superior ou através de sites que abordam o conteúdo. A visualização dos materiais ou jogos construídos está disponível por meio de fotos para observação (PAIM, 2012). 


\section{CONCLUSÃO}

Espera-se que, por meio das práticas relatadas, os alunos não se limitem na confecção dos materiais, mas que sejam motivados a buscar, produzir e compreender conhecimentos matemáticos, desconstruindo paradigmas de que o professor é o único dotado de saber.

As construções podem auxiliar na aprendizagem e no desenvolvimento da criatividade do estudante. E, partindo da ideia de que o IFBA é uma instituição que preza pela qualidade do ensino técnico profissionalizante, é necessário sair do conhecimento matemático repetitivo, tornando-o mais criativo devido ao perfil dos cursos ofertados. Nesse contexto, a criação dos materiais surge como um caminho para a concretização desse processo.

\section{REFERÊNCIAS}

BRASIL. Ministério da Educação e do Desporto. Secretaria de Educação Fundamental. Parâmetros Curriculares Nacionais: matemática. Brasília, DF: MEC, 1997.

Secretaria da Educação Fundamental. Parâmetros Curriculares Nacionais: matemática. Brasília: MEC/SEF, 1998.

BORBA, M. de C.; PENTEADO, M. G. Informática e educação matemática. 4. ed. Belo Horizonte: Autêntica, 2010.

BRAVO, J. A. F. La didáctica de la matematica en la educacion infantil. Pedagogicas, 1995.

DANTE, L. R. Matemática: vivência e construção. 2. ed. São Paulo: Ática, 2005.

FIORENTINI, D.; LORENZATO, S. Investigação em educação matemática. Campinas: Autores Associados, 2009.

FORQUIN, J. C. Escola e cultura: as bases sociais e epistemológicas do conhecimento escolar. Porto Alegre: Artes médicas, 1993.

LOPES, C. S. Apresentação. Revista de Educação Matemática, Campinas-SP, v. 9, n. 9-10, 2004.

LORENZATO, S. O laboratório de ensino de matemática na formação de professores. 2. ed. Campinas, SP: Autores Associados, 2009. 
MORGADO, A. C. et al. Análise combinatória e probabilidade. Rio de Janeiro: Sociedade Brasileira de Matemática, 2004. (Coleção do professor de matemática).

MATOS, J. M. L.; SERRAZINA, M. de L. M. Didática da matemática. Lisboa: Universidade Aberta, 1996. cap. 7. Recursos na aula de Matemática, p. 191-212.

NARDI, R. A pesquisa em ensino de ciências no Brasil: alguns recortes. São Paulo: Escrituras Editoras, 2007.

PAIM. M. A. S. Jogos produzidos pelos alunos do IFBA de Eunápolis - BA, 2012. Disponível em: <http://maspaim.blogspot.com>. Acesso em: 11 abr. 2014.

PAIS, L. C. Intuição, experiência e teoria geométrica. Revista Zetetiké, Campinas - SP, v. 4, n. 6, p. $65-74$, jul/dez. 1996.

SANTANA, E. R. S. Estruturas Aditivas: o suporte didático influencia a aprendizagem do estudante? 2010. 344 f. Tese. (Doutorado em Educação) - Pontifícia Universidade Católica de São Paulo (PUC-São Paulo), São Paulo, 2010. 